\title{
Erratum to: An Investigation into the ASTM E756-05 Test Standard Accuracy on Determining the Damping Properties of Materials in Tension-Compression
}

\section{N. Paimushin ${ }^{a, b}$, V. A. Firsov ${ }^{a,{ }^{*}}$, V. M. Shishkin ${ }^{c}$, V. A. Kostin ${ }^{a}$, and R. Sh. Gimadiev ${ }^{d}$}

${ }^{a}$ Tupolev Kazan National Research Technical University, ul. Karla Marksa 10, Kazan, 420111 Tatarstan, Russia

${ }^{b}$ Kazan (Volga region) Federal University, ul. Kremlevskaya 18, Kazan, 420008 Tatarstan, Russia

${ }^{c}$ Vyatka State University, ul. Moskovskya 36, Kirov, 610000 Russia

${ }^{d}$ Kazan State Power Engineering University, ul. Krasnosel'skaya 51, Kazan, 420066 Tatarstan, Russia

*e-mail:vafirsov_49@mail.ru

Received November 9, 2020

DOI: $10.3103 /$ S1068799820030277

The TeX presentation of formulas was updated in HTML file.

The original article can be found online at https://doi.org/10.3103/S106879982002004X. 